\title{
Immunomodulatory Effects of Supplementation with Extracts from the Marine Brown Alga Eisenia bicyclis on Macrophages
}

\author{
Seung Namkoong ${ }^{1}$, Se-Chan Kang ${ }^{2}$, Hang Do ${ }^{3}$, Ki-Hyo Jang ${ }^{4}$, Seon-A Jang ${ }^{5}$, \\ Myoung-Gun Choung ${ }^{5}$ and Eun-Hwa Sohn ${ }^{5}$ *
}

${ }^{1}$ Department of Physical Therapy, Kangwon National University, Samcheok 245-907, Korea, ${ }^{2}$ Department of Natural Medicine Resources, Semyung University, Jecheon 309-711, Korea, ${ }^{3}$ College of Pharmacy, Sungkyunkwan University, Suwon 440-746, Korea, ${ }^{4}$ Department of Food and Nutrition, ${ }^{5}$ Department of Herbal Medicine Resource, Kangwon National University, Samcheok 245-907, Korea

\begin{abstract}
A large number of edible seaweeds are consumed by the coastal peoples of Asia. Some of them are used in traditional remedies in many parts of the world. In this study we investigated effects of supplementation with ethyl acetate extracts of the brown alga Eisenia bicyclis (EBE) on rat macrophage to evaluate the possibilities as immune-modulators. Twelve male SD rats were divided into two groups and the treatments were as follows: A, no Eisenia bicyclis extract (EBE) intake and distilled water; B, oral supplemented with EBE $200 \mathrm{mg} / \mathrm{kg}$. After 5 weeks of supplementation, rats were sacrificed to assess the effect on peritoneal macrophage functions. We showed no increasing effects on tumoricidal activity, phagocytic activity and NO production in macrophages in EBE supplementation group. However, EBE supplementation suppressed NO-iNOS production and p65 translocation into the nucleus in LPS-stimulated macrophages. Overall, these results suggest that the supplementation of EBE might have an anti-inflammatory effects on NO-iNOS production in macrophages throughout the inhibition of NF-kB activation.
\end{abstract}

Key words - Eisenia bicyclis, iNOS, Macrophage, Nitric oxide, p65

\section{Introduction}

Marine environment has been the source of diverse life forms that produce different biologically active compounds. Metabolites from marine plants have been reported to have outstanding biological activities and profound applications in nutraceuticals, cosmeceuticals, and pharmaceuticals. Eisenia bicyclis is a species of the kelp, a member of the brown algae group of seaweeds, best known for its use in Asia. E. bicyclis is high in calcium, iodine, iron, magnesium, and vitamin $\mathrm{A}$ as well as being a good dietary source for many other minerals. It has been also known that brown algae extracts closely linked to its protective properties against free radical attack and have anti-oxidant effects (Hu et al., 2010; Kang et al., 2004). They contain the storage polysaccharide laminarin, alginate, fucoxanthin and fucoidan with immunological activities (Joe et al., 2006).

*Corresponding author. E-mail : ehson@kangwon.ac.kr
Macrophage have been shown to be an important component of host defense against bacterial infection, and cancer (Hahn and Kaufmann, 1981; Verstovsek et al., 1992). Large pools of macrophages, thought to be of mononuclear phagocyte origin, are located throughout the body and historically have been identified by different names including peritoneum (peritoneal macrophages), brain (microglia), bone (osteoclast) and liver (Kupffer cells). In murine/rat peritoneal system, four general stages of activation have been defined and cells in these stages are called resident, inflammatory (or responsive), primed and fully activated macrophages (Klimp et al., 2002). When exposed in vitro LPS and (or) IFN- $\gamma$, inflammatory macrophages can develop the capacity to kill tumor cells, and these stages are called fully activated macrophages. During develop other stages, macrophages increase the phagocytic activity and release various materials such as cytokines and reactive intermediates and then carry out nonspecific immune responses (Adams and Hamilton, 1981; Nathan, 1987). Especially, there has been great interest in reactive nitrogen 
intermediate, nitric oxide (NO) which is considered to be a central molecule in the regulation of the immune response to tumors and inflammation because of its cytotoxic effects (Fidler and Kleinerman, 1983; Keller and Keist, 1989; Bredt and Snyder, 1994).

Recently, the biological and pharmacological properties of the brown algae have begun to receive more attention in the scientific community and have become a very important research focal point. However, the immune-modulatory effects of Eisenia bicyclis extracts (EBE) on primary macrophages are still unknown. In our previous study, we determined the concentration range of EBE in rats. No toxic effects of the EBE supplementation were observed to any of organs in rats. Here we examined the immune-modulatory effects of the supplementation of EBE on the effects of macrophagemediated tumoricidal activity, phagocytic activity, and the production of intermediate regulatory molecule, NO-iNOS in rats. Additionally, we determined the regulation of NO-iNOS in LPS-stimulated macrophages and the translocation of NF$\kappa \mathrm{B}$ (p65) which is activated during inflammation, in LPStreated macrophages to validate the anti-inflammatory effects of EBE.

\section{Materials and Methods}

\section{Preparation of ethyl acetate extract}

The fronds of E. bicyclis were harvested from the coast of Ulleung Island, East Sea, South Korea in June 2009. Fresh $E$. bicyclis was washed three times with tap water to remove salt and impurities, then dried at room temperature for 3 days and stored at $-20^{\circ} \mathrm{C}$. The dried samples were homogenized, using a grinder, before extraction. Dried E. bicyclis powder $(10 \mathrm{~g})$ was dissolved in $70 \% \mathrm{MeOH}(200 \mathrm{ml})$ and shaken for $24 \mathrm{~h}$. After the extraction, the supernatant was recovered by centrifugation $(7000 \mathrm{rpm})$ at $4{ }^{\circ} \mathrm{C}$ for $30 \mathrm{~min}$ and the methanol was evaporated using a rotary vacuum evaporator (Tokyo Rikakikai Co. Ltd., Tokyo, Japan). The extract powder was suspended in distilled water and then partitioned with n-hexane, methylene chloride, ethyl acetate (EtOAc) in sequence. EtOAc fraction was evaporated and kept on $-20^{\circ} \mathrm{C}$ until used. We used the layer of EtOAc extracts from EBE because it showed significantly inhibitory effect on NO production induced by LPS.

\section{Experimental animals}

Male SD rats were purchased from Joongang Experimental Animal Co. (Seoul, Korea) at 5 weeks of age, and the six animals were housed together in one cage in controlled environment under a light-dark cycle (lights on at 19:00 and off at 7:00). The experimental procedures were conducted according to the Code of Ethics for Animal Experimentation of Semyung University. After 1 week acclimation, all rats were randomly divided into two groups and the treatments were as follows: A, no EBE intake and distilled water ; B, oral supplemented with EBE $200 \mathrm{mg} / \mathrm{kg}$. After 5 weeks for oral supplementation, rats were sacrificed to assess the effects on peritoneal macrophages.

\section{Chemicals}

Unless stated otherwise, all chemicals were purchased from the Sigma-Aldrich (St Louis, MO). The RPMI 1640 medium and fetal bovine serum (FBS) were purchased from GIBCO (Grand Island, NY). The XTT \{2,3-Bis(2-methoxy4-nitro-5-sulfophenyl)-2H-tetrazolium-5-carboxanilide inner salt\} cell viability assay kit was purchased from WelGENE (Daegu, Korea). All the tissue culture reagents and ethyl acetate fraction from E. bicyclis were assayed for any endotoxin contamination using the Limulus lysate test (E-Toxate, Sigma), and the endotoxin levels were found to be $<10 \mathrm{pg} / \mathrm{ml}$.

\section{Macrophage-mediated tumoricidal activity}

The assay for macrophage-mediated tumoricidal activity was determined using A modification of the method reported by Mosmann (1983). Briefly, macrophages $\left(1 \times 10^{5}\right.$ cells/well $)$ from rats first incubated in either medium alone or in medium supplemented with LPS for $24 \mathrm{~h}$ in 96-well plates. Macrophages were washed with RPMI-FBS to remove LPS and then co-incubated with B16 melanoma cells $\left(1 \times 10^{4}\right.$ cells/ well; effector : target cell ratio of 10:1). After $24 \mathrm{~h}$, plates were stained with crystal violet containing $10 \%$ formaldehyde for $15 \mathrm{~min}$. Absorbance of each well at $540 \mathrm{~nm}$ was determined by using Molecular Devices microplate reader (Menlo Park, CA). Cytotoxic activity is expressed as the percentage of 
tumor cytotoxicity by the following formula : [1-\{O.D. of (target cells + macrophages) - O.D. of macrophages $\} / O . D$. of target cells] $\times 100$

O.D. of target cells is the optical density of B16 melanoma cells and OD of macrophages is the optical density of macrophages.

\section{NBT assay for phagocytosis}

Phagocytosis was measured by nitro blue tetrazolium (NBT) reduction assay (Okimura et al., 1986). Peritoneal macrophages were seeded in 96 -well plates at a density $5 \times 10^{4}$ cells per well and cultured for $24 \mathrm{~h}$. The cultured media was then removed and $50 \mu \mathrm{l}$ of $5 \times 10^{6}$ particles $/ \mathrm{ml}$ zymosan and $0.6 \mathrm{mg} / \mathrm{ml} \mathrm{NBT}$ was added into each well. After an additional incubation for $1 \mathrm{~h}$, wells were washed with cold D-PBS two times and the optical density of reduction product of NBT, a purple insoluble formazan, was determined at $540 \mathrm{~nm}$ using a microplate reader. It was not required to solubilize the formazan before taking the measurement of absorbance.

\section{Nitrite determination}

Peritoneal macrophages were incubated in either medium alone or in medium supplemented with LPS for $24 \mathrm{~h}$ and the accumulation of nitrite in culture supernatants was measured using the assay system described by Ding et al., (1988). 100 $\mu \mathrm{L}$ aliquots of culture supernatants were mixed with an equal volume of Griess reagent (mixture at 1:1 of naphthylethylenediamine dihydrochloride and $1 \%$ sulphanilamide in $5 \%$ $\mathrm{H}_{3} \mathrm{PO}_{4}$ ) and incubated at room temperature for $10 \mathrm{~min}$. Nitrite concentration was calculated from a $\mathrm{NaNO}_{2}$ standard curve.

\section{Westem blot analysis for iNOS gene expression}

The amount of iNOS was measured by Western blot analysis. After $24 \mathrm{~h}$ of incubation with or without LPS in 6-well plates, the macrophages were lysed in RIPA buffer (50 mM Tris- $\mathrm{HCl}, \mathrm{pH} 8.0,150 \mathrm{mM} \mathrm{NaCl}, 1 \%$ Nonidet-P40, $0.5 \%$ deoxycholic acid, $0.1 \%$ sodium dodecyl sulfate). The protein concentrations were measured using the DC Protein Assay (Bio-Rad Laboratories, Hercules, CA). Twenty micrograms of each sample were electrophoresed on $10 \%$ SDS-PAGE gels and transferred to Hybond-ECL nitrocellulose membranes (Amersham Biosciences, Piscataway, NJ). The membranes were blocked with 5\% skim milk in Tris-buffered saline/ Tween-20 for $1 \mathrm{~h}$. The membranes were incubated with primary antibody against iNOS for $24 \mathrm{~h}$. They were then washed with Tris-buffered saline/Tween-20 once for $15 \mathrm{~min}$ and three times for $5 \mathrm{~min}$, and were incubated with secondary HRP-conjugated anti-rabbit antibody for $1 \mathrm{~h}$. The membranes were then washed again as described above. Autoradiography was carried out using an enhanced chemiluminescence kit (Amersham Bioscience, Piscataway, NJ).

\section{Determinant of translocation of $\mathbf{p 6 5}$}

Nuclear extracts were prepared. In brief, peritoneal macrophages $\left(5 \times 10^{7}\right.$ cells $\left./ \mathrm{ml}\right)$ were plated into $100-\mathrm{mm}$ dishes and treated with LPS $(10 \mu \mathrm{g} / \mathrm{ml})$ for $4 \mathrm{~h}$. The harvested cells were resuspended in $25 \mu \mathrm{lml}$ of buffer A (10 $\mathrm{mM}$ HEPES at $\mathrm{pH} 7.5,1.5 \mathrm{mM} \mathrm{MgCl} 2,10 \mathrm{mM} \mathrm{KCl}, 1 \mathrm{mM}$ DDT, $0.1 \%$ NP-40, $0.2 \mathrm{mM}$ PMSF). The cells were lysed on ice for $15 \mathrm{~min}$, and centrifuged $\left(5,000 \mathrm{~g}, 5 \mathrm{~min}, 4^{\circ} \mathrm{C}\right)$. The nucleic pellet was washed with buffer A lacking NP-40, and resuspended in $0.025 \mathrm{ml}$ of buffer C (20 mM HEPES, pH 7.5, $25 \%$ glycerol, $0.42 \mathrm{M} \mathrm{NaCl}, 0.2 \mathrm{mM}$ EDTA, $1.5 \mathrm{mM} \mathrm{MgCl}_{2}$, $1 \mathrm{mM}$ DDT, $0.2 \mathrm{mM}$ PMSF). After incubation on ice for $30 \mathrm{~min}$, nuclear debris was spin down $\left(13,000 \mathrm{~g}, 10 \mathrm{~min}, 4^{\circ} \mathrm{C}\right)$. The supernatant was collected as nuclear extracts. The protein concentration was measured using a protein assay kit.

\section{Statistical analysis}

Each experiment was repeated at least three times, and the results of one representative experiment are shown. The results were expressed as means \pm S.E.M. and analyzed via ANOVA. The significant values are represented by an asterisk $\left({ }^{*}, \#<0.05\right.$ and $\left.{ }^{* *}, \# \#<0.01\right)$.

\section{Results and Discussion}

The immune system may play an important role in occurring various disease and the changes in the immune status are associated with treatment of various immune-modulators (Speziali et al., 2009). This study examined the immunemodulatory (stimulatory/inhibitory) effects of EBE supplementation on the functions of peritoneal macrophages in rats. Phagocytosis, tumoricidal activities and inflammatory responses 
(a)

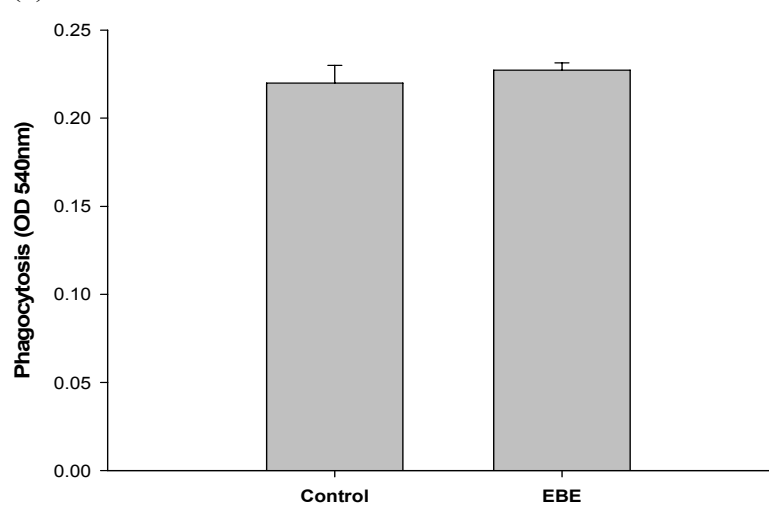

(b)

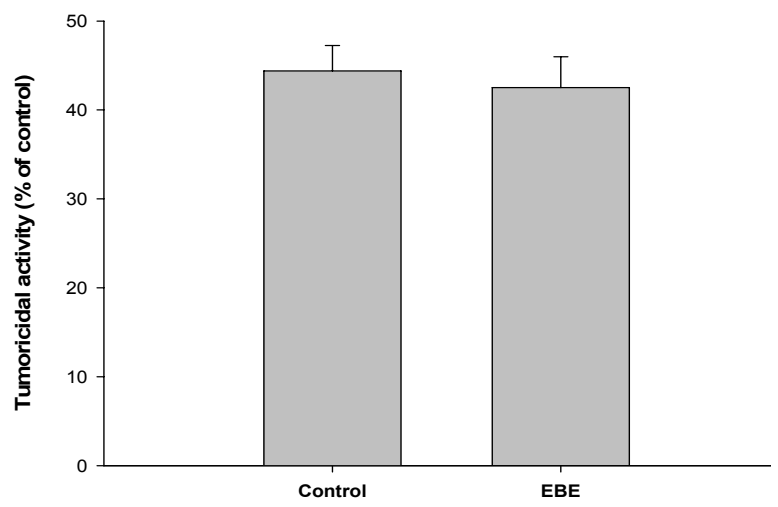

(c)

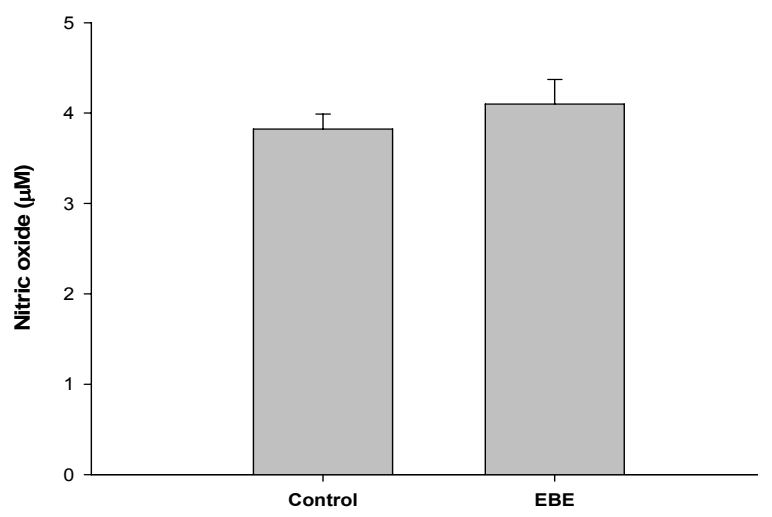

Fig. 1. (A) The effects of the supplementation of EBE (200 $\mathrm{mg} / \mathrm{kg}$ ) on the phagocytic activity, (B) macrophage-mediated tumoricidal activity and $(\mathrm{C})$ the production of $\mathrm{NO}$ by peritoneal macrophages. The peritoneal macrophages $\left(1 \times 10^{5}\right.$ cell/well) from rats were cultured $24 \mathrm{~h}$ and/or co-cultured with B16 tumor target cells (effector: target $=10: 1$ ). These results are means \pm S.E.M of quintuplicates from a representative experiment.

are the immune-modulatory activities of macrophages, which is responsible for a diverse range of antimicrobial- and cytotoxic- activities, including respiratory burst, secretion of inflammatory mediators and antigen presentation. Here, we examined the immune-stimulating effects such as phagocytosis and tumoricidal activities and the immune-inhibitory such as anti-inflammatory effects of EBE supplementation on macrophages in rats.

As shown in Figure 1A and B, EBE have no significantly effects of the phagocytic and tumoricidal activites in peritoneal macrophages. Our data showed that EBE has no immune-stimulatory effects on macrophage functions in rats. Nitric oxide, one of the smallest biological molecular mediators, plays important roles in many different aspects of mammalian biology, such as vascular relaxation, neurotransmission, platelet aggregation and immune regulation (Hemmi and Ishida, 1980; Hibbs et al., 1998). Since NO is related to cytostatic or cytotoxic function of macrophages against a variety of tumors (Keller et al., 1990; Hibbs et al., 1987), we also examined the effects of EBE on NO production from macrophages in rats. As shown in Figure 1C, the supplementation of EBE also no increasing effects of NO production.

It is known that inducible nitrogen synthase (iNOS) is expressed in many different cell types and increased iNOS enzymes produces high levels of nitric oxide. NO was produced in high amounts by iNOS in activated macrophages, and then excessive formation of NO plays a key role in inflammatory response of macrophages. Therefore, overactivated macrophages have been known to produce various pro-inflammatory cytokines such as TNF- $\alpha$, IL-1 $\beta$, IL-6 and NO-iNOS that are plays a key roles in inflammatory response. Thus, we also determine the inhibitory effect of EBE on NO-iNOS production in LPS-stimulated macrophages. As shown in Figure 2, the treatment of EBE decreased NO-iNOS production in LPS-stimulated inflammatory macrophages.

In our study, it showed discriminal effects of EBE alone groups in NO (Fig. 1C) and iNOS (Fig. 2B, 4 lane) production. It seems that EBE increased iNOS gene transcriptional and translational expression, but the amounts of NO which extracellular releasing form is not enough to detect by Griess reagent assay. Therefore we concluded that EBE supplementation has increasing effects of iNOS expression in macrophages and inhibitory effects of iNOS expression in 
(a)

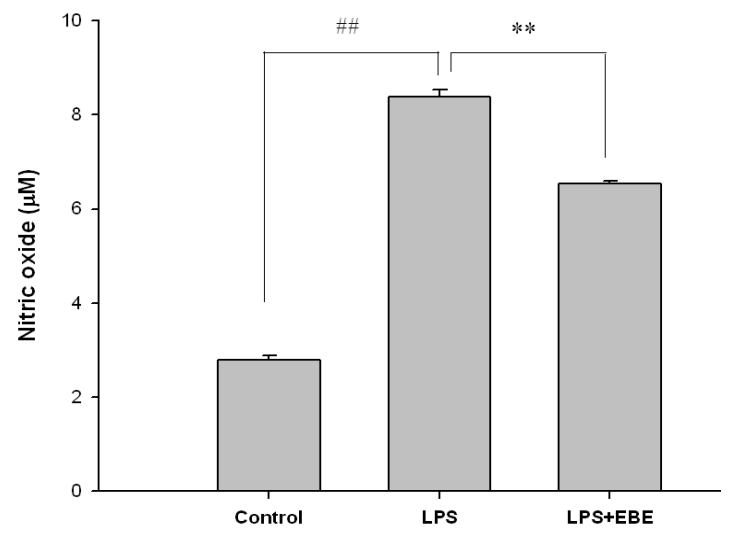

(b)

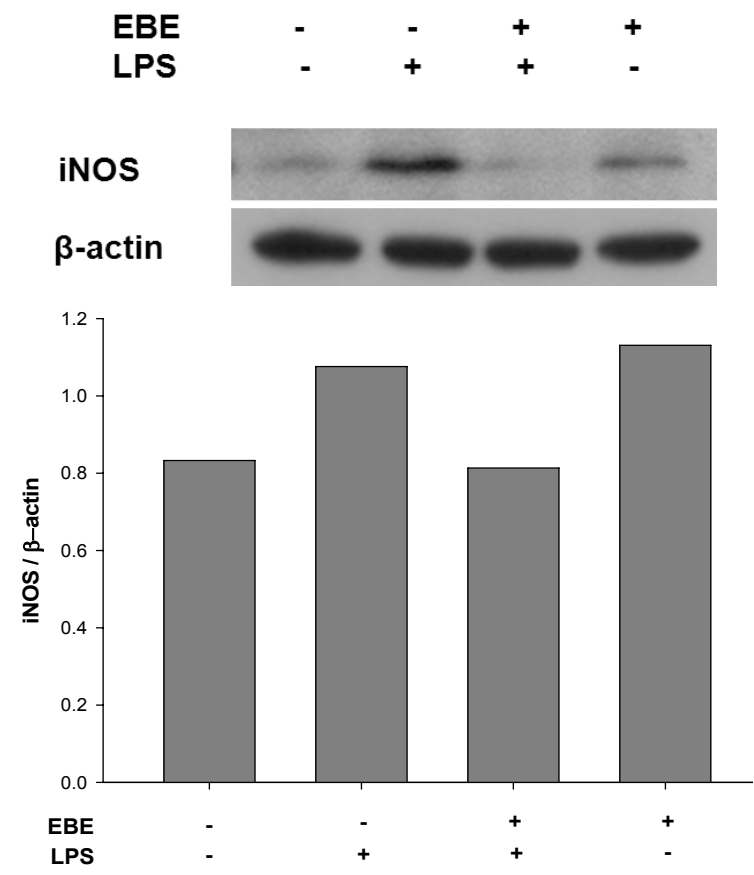

Fig. 2. The inhibitory effects of EBE (200 mg/kg) supplementation on the production of nitrite $(\mathrm{A})$ and the expression of iNOS in LPS-induced peritoneal macrophages (B). LPS $(10 \mu \mathrm{g} / \mathrm{ml})$ were treated to macrophages with or without LPS for $20 \mathrm{~h}$. Each bar shows the means \pm S.E.M of four independent experiments. ${ }^{\#} P<0.01$ : significantly different from the untreated control group. ${ }^{* *} P<0.01$ : significantly different from the LPS treated group.

LPS-stimulated macrophages. At present time the mechanisms by which EBE has a biphasic response to macrophages are not clear and further study should be needed.

$\mathrm{NF}-\kappa \mathrm{B}$ (p65) translocation is regarded as a important processes in the activation of the innate and acquired immune

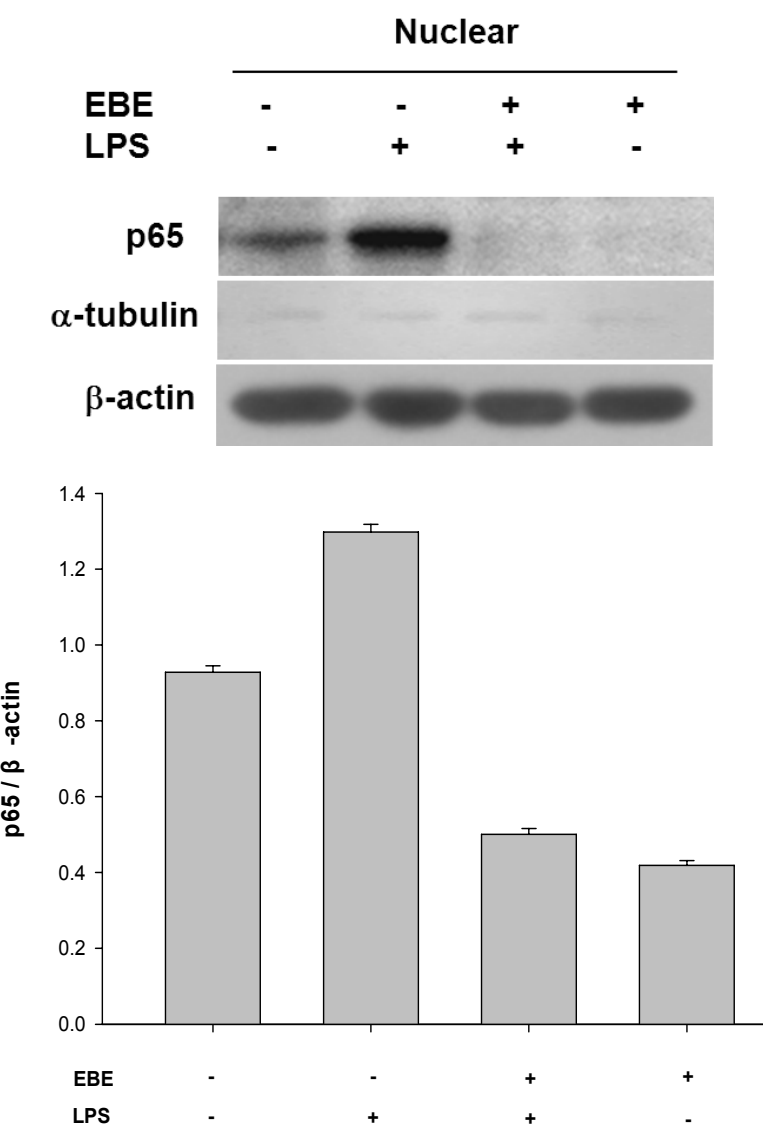

Fig. 3. The inhibitory effects of $\operatorname{EBE}(200 \mathrm{mg} / \mathrm{kg})$ supplementation on NF- $\kappa \mathrm{B}(\mathrm{p} 65)$ activation in LPS-induced peritoneal macrophages. LPS $(10 \mu \mathrm{g} / \mathrm{ml})$ were treated to macrophages with or without LPS for $4 \mathrm{~h}$. After isolation of nuclear fraction, the translocation of NF- $\mathrm{BB}$ (p65) was assessed by Western blotting described in methods.

responses and chronic inflammation (Karin et al., 2002; Barnes et al., 1997). NF- $\mathrm{KB}$ is also known to be a critical transcription factor that regulates inflammatory responses (Yang et al., 1998; Barnes et al., 2008). In our study, increased expression of NF- $\mathrm{KB}$ (p65) was observed in the nucleus after treatment with LPS for $4 \mathrm{~h}$ (Figure 3 ). The relative intensity of NF- $\kappa \mathrm{B}$ (p65) translocation in the nucleus was increased to $39.8 \%$ in the presence of LPS compared with the absence of LPS in the control rats. In contrast, the relative intensity of NF- $\kappa \mathrm{B}$ (p65) translocation in the nucleus was decreased considerably, to $61.4 \%$ in EBE supplementation rats in the presence of LPS. These data demonstrate that EBE attenuated NF- $\mathrm{KB}$ activation in LPS-stimulated macrophages. These results suggest that EBE inhibits the over-production 
of NO-iNOS by downregulating NF- $\mathrm{B}$ activation in LPSstimulated macrophages and supports the hypothesis that EBE may have beneficial effects in the treatment of various inflammatory disease.

\section{Literature Cited}

Adams, D.O. and T.A. Hamilton. 1984. The cell biology of macrophage activation. Annu. Rev. Immunol. 2:283-318.

Barnes, P.J. 2008. Role of GATA-3 in allergic diseases. Curr. Mol. Med. 8:330-334.

Bredt, D.S. and S.H. Snyder. 1994. Nitric oxide: a physiologic messenger molecule. Annu. Rev. Biochem. 63:175-195.

Ding, A.H., C.F. Nathan and D.J. Stuer. 1988. Release of reactive nitrogen intermediates and reactive oxygen intermediates from mouse peritoneal macrophages. Comparison of activating cytokines and evidence for independent production. J. Immunol. 141:2407-2412.

Fidler, I.J. and E.S. Kleinerman. 1983. Therapy of cancer metastasis by systemic activation of macrophages: from the bench to the clinic. Res. Immunol. 144:284-287.

Hahn, H. and S.H. Kaufmann. 1981. The role of cell mediated immunity in bacterial infections. Rev. Infect. Dis. 3:12211250.

Hemmi, H. and N. Ishida. 1980. The immune response of splenic lymphocytes after cimicifugoside treatment in vitro and pretreatment in vivo. J. Pharmacobiodyn. 3:643-648.

Hibbs, J.B. Jr., R.R. Taintor and Z. Vavrin. 1987. Macrophage cytotoxicity: role for L-arginine deaminase and imino nitrogen oxidation to nitrite. Science 235:473-476.

Hibbs, J.B. Jr,, R.R. Taintor, Z. Vavrin and E.M. Rachlin. 1998 Nitric oxide: a cytotoxic activated macrophage effector molecule. Biochem. Biophys. Res. Commun. 157:87-94.

Hu, T., D. Liu, Y. Chen, J. Wu and S. Wang. 2010. Antioxidant activity of sulfated polysaccharide fractions extracted from Undaria pinnitafida in vitro. Int. J. Biol. Macromol. 46: 193-198.

Joe, M.J., S.N. Kim, H.Y. Choi, W.S. Shin, G.M. Park, D.W. Kang and Y.K. Kim. 2006. The inhibitory effects of eckol and dieckol from Ecklonia stolonifera on the expression of matrix metalloproteinase-1 in human dermal fibroblasts. Biol. Pharm. Bull. 29:1735-1739.
Kang, K., H. J. Hwang, D. H. Hong, Y. Park, S. H. Kim, B. H. Lee and H. C. Shin. 2004. Antioxidant and antiinflammatory activities of ventol, a phlorotannin-rich natural agent derived from Ecklonia cava, and its effect on proteoglycan degradation in cartilage explant culture. Res. Commun. Mol. Pathol. Pharmacol. 115-116:77-95.

Karin, M. and A. Lin. 2002. NF- $\mathrm{KB}$ at the crossroads of life and death. Nat. Immunol. 3:221-227.

Keller, R. and R. Keist. 1989. Abilities of activated macrophages to manifest tumoricidal activity and to generate reactive nitrogen intermediates: a comparative study in vitro and ex vivo. Biochem. Biophys. Res. Commun. 164:968-973.

Keller, R., R. Keist and K. Frei. 1990. Lymphokines and bacteria that induce tumoricidal activity, trigger a different secretory response in macrophages. Eur. J. Immunol. 20: 695-698.

Klimp, A.H., E.G. de Vries, G.L. Scherphof and T. Daemen. 2002. A potential role of macrophage activation in the treatment of cancer. Crit. Rev. Oncol. Hematol. 44:143-161.

Mosmann, T. 1983. Rapid colorimetric assay for cellular growth and survival: application to proliferation and cytotoxicity assays. J. Immunol. Methods 65:55-63.

Nathan, C.F. 1987. Secretory products of macrophages. J. Clin. Invest. 79:319-326.

Okimura, T., M. Ogawa and T. Yamauchi. 1986. Stress and immune responses. III. Effect of resistant stress on delayed type hypersensitivity (DTH) response, natural killer (NK) activity and phagocytosis in mice. Japanese J. Pharmacol. 41: 229-235.

Speziali, E., A.F. Santiago, R.M. Fernandes, N.M. Vaz, J.S. Menezes and A.M. Faria. 2009. Specific immune responses but not basal functions of $\mathrm{B}$ and $\mathrm{T}$ cells are impaired in aged mice. Cell. Immunol. 256:1-5.

Verstovsek, S., D.L. Maccubbin, M.J. Ehrke and E. Mihich. 1992. Tumoricidal activation of murine resident peritoneal macrophages by interleukin-2 and tumor necrosis factor. Cancer Res. 52:3880-3885.

Yang, L., L. Cohn, D.H. Zhang, R. Homer, A. Ray and P. Ray P. 1998. Essential role of nuclear factor- $\mathrm{KB}$ in the induction of eosinophilia in allergic airway inflammation. J. Exp. Med. 188:1739-1750.

(Received 19 May 2011 ; Accepted 9 June 2011) 